\title{
Volumetric measurements of the subcortical structures of healthy adult brains in the Turkish population
}

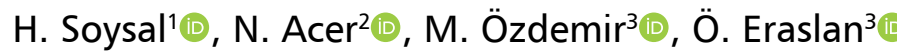 \\ ${ }^{1}$ Department of Anatomy, Faculty of Dentistry, Ankara Yıldırım Beyazıt University, Ankara, Turkey \\ 2Department of Anatomy, Faculty of Medicine, Arel University, Istanbul, Turkey \\ ${ }^{3}$ Department of Radiology, Dışkapı Yıldırım Beyazıt Health Application and Research Centre, \\ Medical Sciences University, Ankara, Turkey
}

[Received: 4 January 2021; Accepted: 5 March 2021; Early publication date: 22 March 2021]

Background: The interest in the morphological development of brain structures during childhood and adolescence arises from discussions on subcortical anomalies and sexual dimorphism, from adolescent changes in cognitive functions supported by cortical and subcortical structures to a wide range of childhood neuropsychiatric diseases. This study aims to investigate the subcortical structures regarding age/gender changes in the healthy adult human brain using web-based volBrain.

Materials and methods: In this study, 303 normal healthy adults (males and females) were examined using a $1.5 \mathrm{~T}$ unit with a 20-channel head coil.

Results: The volumes of white matter, grey matter, total brain, cerebrospinal fluid, and total intracranial volume were significantly higher in males than those in females. Our analysis revealed a significantly larger accumbens volume in females. With the age of less than or equal to 50 years, older males were found to have higher total lateral ventricle, putamen, thalamus, amygdala, cerebrum, white matter and grey matter volumes than females. In the age group of 50 years and older mean total volumes of thalamus, globus pallidus and accumbens were higher in females than those in males. Right hemisphere volumes in younger and older age groups were higher except for caudate volume in the older age group; the mean of caudate was significantly higher in females than those in males.

Conclusions: These conclusions might be important for the explanation of the effects of gender and age in cross-sectional structural magnetic resonance imaging studies. Also, knowing the volume changes of the subcortical structures can provide convenience about the prevention, diagnosis, and treatment of various neuromental disorders. (Folia Morphol 2022; 81, 2: 294-306)

Key words: cortical volume, subcortical nuclei, sex differences, magnetic resonance imaging, healthy adult brain, volBrain 


\section{INTRODUCTION}

Magnetic resonance imaging (MRI) allows examination of brain parts in a noninvasive way and in vivo. This is significant in studying the pathogenesis and pathophysiology of neurological disturbances [18]. There are many volumetric brain studies that show neuroanatomical disorders in MRI [14].

Previous studies have reported an increase in the volume of basal ganglia in schizophrenia [8]. However, new volumetric studies have reported a decrease in the volume of basal ganglia such as putamen, caudate, thalamus [17, 19, 32].

Autopsy studies show that brain weight decreases by at least $10 \%$ in men and women between the ages of $25-75$, also in the age range of $50-75$, the volume decreases by about $2 \%$ every decade [4].

The brain differs depending on age/gender. Variety can be measured in vivo with MRI. The volumetric MRI analyses indicate that age-associated volume reductions have been reported especially for basal ganglia such as the thalamus, caudate, putamen, globus pallidus (GP) $[2,22]$.

There are many studies about basal grey structures' volume depending on age and gender using different methods [17, 24, 25, 31, 32].

Studies on volume indicated a negative proportion among age, sex, and basal ganglia volumes [1]. Male caudate volume is higher than female [11].

There are many reports to support the effect of subcortical volumes in gender-specific neuropsychiatric disturbances (e.g. autism, schizophrenia, Parkinson's disease, attention deficit hyperactivity disorder, and addictions) [5, 35, 38].

Recently, different techniques have been studied for automatic or semi-automatic segmentations of subcortical structures, such as software for analysis and visualisation of functional magnetic resonance neuroimages [10], BrainVoyager [15], FreeSurfer [12, 41], Mristudio $[21,26]$ and statistical parametric maps [2] — software used to analyse the structural features of the human brain. volBrain is an automated method where the observer can perform fully automatic segmentation using a web-based application. Recently, volBrain has been used for a neuroimaging study of MRI data [23].

volBrain is an automatic and sturdy quantitative analysis system that also gives a result in a short time. In our study, we share the results of the MRI study on the effects of age on subcortical structures using volBrain. All participants consisted of 303 subjects aged from 12 to 84 years (113 men, 190 women).

\section{MATERIALS AND METHODS}

\section{Participants}

All participants provided written informed consent for the relevant studies and ethical approval was obtained from the Dışkapı Training and Research Hospital Clinical Research Ethics Committee. Research was conducted on human participants. All procedures performed in this study comply with the ethical standards of the institution. Approval was obtained from the Ethics Committee of Dışkapı Yıldırım Beyazıt Training and Research Hospital, indicating that the MRI used in the study were ethically and scientifically safe (Ethics Committee's decision date: 06.08.2018; Decision number: 53/05).

The study group consisted of healthy volunteers with no history of surgery or trauma of the brain, neurological or psychiatric disease, or substance abuse.

A mini-score assessment was performed in order to rule out psychiatric disease as well as cognitive impairment. Finally, our study group consisted of 303 participants. Participants included 113 men and 190 women with a mean age of 49 years (range: 12-84) and 42.5 years (range: 11-82), respectively. Informed consent was obtained from parent and/ or legal guardian for human participants under the age of 18. Children under the age of 18 came to the hospital with their parents, and brain MRI radiographs were taken after obtaining informed consent from the parents.

\section{MRI protocol and segmentation method}

Magnetic resonance imaging of the subcortical structures was acquired on a 1.5 T unit (Magnetom Aera, Siemens, Erlangen, Germany) with a 20-channel head coil. The subcortical volumetric assessment was conducted on sagittal oblique T1-weighted images obtained perpendicular to the long axis of the subcortical structures. A magnetisation prepared rapid acquisition gradient echo sequence (MP-RAGE) was used with the following parameters: repetition time $=2400 \mathrm{~ms}$, echo time $=3.54 \mathrm{~ms}$, field of view $=240 \mathrm{~mm}$, slice thickness $=1.2 \mathrm{~mm}$, voxel size $=1.3 \times 1.3 \times 1.2 \mathrm{~mm}$.

Magnetic resonance imaging data processing and subcortical volumetric analyses were performed using volBrain (v.1.0, http://volbr ain.upv.es), a free online MRI brain volumetry system. volBrain is a fully automated segmentation technique of which the algorithm is based on multi-atlas patch-based label fusion segmentation technology (Figs. 1, 2) [9, 23]. 

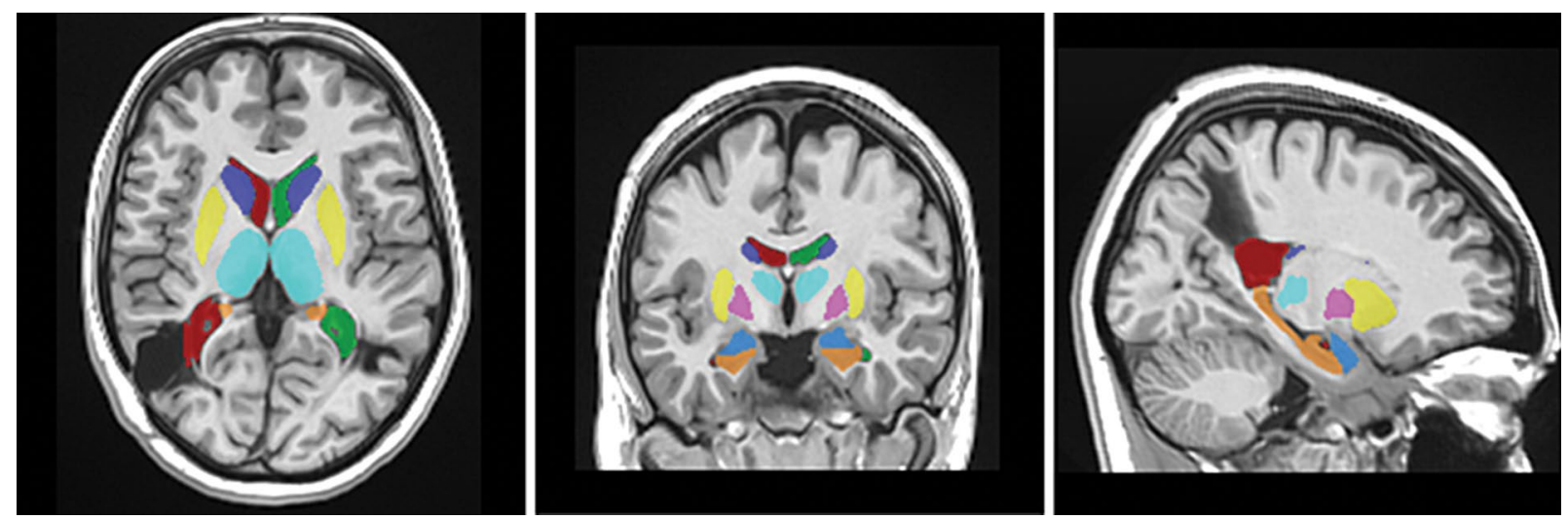

Figure 1. Fully-automated subcortical segmentation by volBrain.
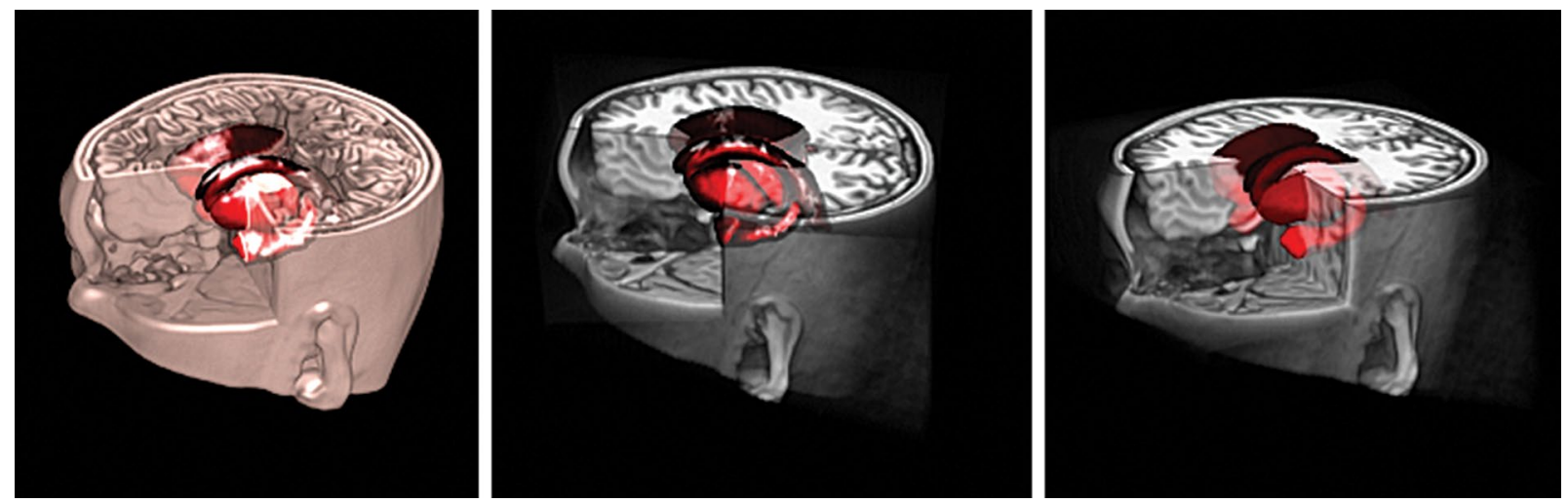

Figure 2. Three-dimensional visualisation of volBrain data.

\section{Statistical analysis}

The distributions of age and volume measurements were examined by the Shapiro-Wilk's test and normality plots. All continuous variables were reported as median (range). Total and side volumes were also summarized by mean \pm standard deviation (mean \pm SD).

Genders were compared by the Mann-Whitney $\mathrm{U}$ test for age and volume measurements. Additionally, independent samples t-test was performed in comparison between males and females for normally distributed volumes in the oldest age group. The adjustment of age and total intracranial volume (TIC) was also applied for the comparisons of volumes by general linear model procedure. Intracranial volume (ICV) was used as a covariate to compare the volumes of males and females in age groups. The volumes of the left and right hemisphere of the brain were compared by the Wilcoxon test in males and females, separately. A p-value $<0.05$ was considered as statistically significant.
Statistical analyses were performed via IBM SPSS Statistics 22.0 (IBM Corp. Released 2013. IBM SPSS Statistics for Windows, Version 22.0. Armonk, NY: IBM Corp.).

\section{RESULTS}

\section{Global effect of aging}

The median age of men and women was 49 (range: 12-84) and 42.5 years (range: 11-82), respectively. There was no significant difference between men and women by age $(p=0.071)$. The volumes of white matter (WM), grey matter (GM), total brain (TB), cerebrospinal fluid (CF), and TIC were significantly higher in males than females ( $p<0.001$ for all, Table 1 ).

\section{Gender differences for subcortical nucleuses}

The univariate analysis revealed that the volumes of lateral ventricle (LV), putamen, GP, amygdala, cerebrum, WM, and GM were significantly higher in men ( $p<0.05$, Table 2). When the adjustment according 
Table 1. Age and volume distributions in males and females

\begin{tabular}{lccc}
\hline & Male $(\mathbf{n}=\mathbf{1 1 3})$ & Female $(\mathbf{n}=\mathbf{1 9 0})$ & Total $(\mathbf{n}=\mathbf{3 0 3})$ \\
\hline Age $[$ years] & $49(12-84)$ & $42.5(11-82)$ & $45(11-84)$ \\
Volumes $\left[\mathrm{mm}^{3}\right]^{* *}:$ & & & \\
$\quad$ White matter & $530.16(317.29-785.44)$ & $477.73(215.18-1,027.72)$ & $493.35(215.18-1,027.72)$ \\
Gary matter & $719.07(401.78-929.19)$ & $661.45(242.56-903.33)$ & $681.21(242.56-929.19)$ \\
Total brain & $1,241.83(862.18-1,548.87)$ & $1,151.11(644.70-1,475.80)$ & $1,183.99(644.70-1,548.87)$ \\
Cerebrospinal fluid & $242.30(101.99-518.05)$ & $178.46(62.79-596.96)$ & $195.09(62.79-596.96)$ \\
Total intracranial & $1,494.78(1,146.07-1,866.76)$ & $1,329.50(941.06-1653.44)$ & $1,374.51(941.06-1,866.76)$ \\
\hline
\end{tabular}

Data are shown as median (minimum-maximum); ${ }^{*} p=0.071,{ }^{* *} p<0.001$

Table 2. Distribution of subcortical volumes in males and females

\begin{tabular}{|c|c|c|c|c|c|c|}
\hline & \multicolumn{2}{|r|}{ Male } & \multicolumn{2}{|r|}{ Female } & \multirow[t]{2}{*}{ P-value } & \multirow{2}{*}{$\begin{array}{c}\text { Adj. } \\
\text { p-value }\end{array}$} \\
\hline & Mean \pm SD & Median (min-max) & Mean \pm SD & Median (min-max) & & \\
\hline LV & $23.08 \pm 18.04$ & $16.96(2.20-83.92)$ & $13.48 \pm 15.88$ & $9.75(2.09-149.93)$ & $<0.001$ & 0.260 \\
\hline Caudate & $7.01 \pm 1.54$ & $6.81(4.39-18.92)$ & $6.82 \pm 0.97$ & $6.75(4.41-11.55)$ & 0.354 & 0.265 \\
\hline Putamen & $8.49 \pm 1.69$ & $8.31(5.52-21.97)$ & $8.07 \pm 1.08$ & $8.11(3.60-13.41)$ & 0.012 & 0.785 \\
\hline Thalamus & $11.04 \pm 2.13$ & $11.07(6.86-27.04)$ & $10.69 \pm 1.28$ & $10.75(6.51-14.64)$ & 0.178 & 0.293 \\
\hline GP & $2.39 \pm 0.44$ & $2.37(1.27-5.10)$ & $2.26 \pm 0.35$ & $2.27(0.68-3.14)$ & 0.021 & 0.276 \\
\hline Amygdala & $1.77 \pm 0.34$ & $1.78(0.44-2.60)$ & $1.64 \pm 0.31$ & $1.68(0.04-2.65)$ & $<0.001$ & 0.635 \\
\hline Accumbens & $0.66 \pm 0.18$ & $0.67(0.04-1.08)$ & $0.68 \pm 0.16$ & $0.68(0.07-1.11)$ & 0.347 & $<0.001$ \\
\hline Cerebrum & $1,088.94 \pm 119.37$ & $1,090.01(746.69-1,345.42)$ & $998.02 \pm 106.54$ & $1,001.35(607.16-1,308.94)$ & $<0.001$ & 0.133 \\
\hline WM & $474.60 \pm 73.11$ & $484.08(288.09-701.24)$ & $433.93 \pm 72.21$ & 429.58 (170.51-879.07) & $<0.001$ & 0.144 \\
\hline GM & $614.34 \pm 74.20$ & $609.66(348.86-814.31)$ & $564.09 \pm 70.74$ & 555.75 (214.27-795.99) & $<0.001$ & 0.830 \\
\hline
\end{tabular}

SD — standard deviation; LV — lateral ventricle; GP — globus pallidus; GM — grey matter; WM — white matter; Adj. p-value — p-value after total intracranial volume and age adjustment

to the age and TIC was applied, the only significant difference was in accumbens volume between males and females; it was found to be higher in women compared to males ( $p<0.001$ ) (Fig. 3).

\section{Side differences}

The right brain volumes were significantly lower for LV, putamen, accumbens and WM, and higher for caudate than those of the left brain in males. The right brain volumes were significantly lower for LV, putamen, thalamus, GP, amygdala, accumbens and WM, and higher for caudate and GM than those of the left volumes in females (Fig. 4, Table 3).

\section{Gender differences in the younger age group}

When the individuals with age less than or equal to 50 years were examined, males were found to have higher total LV, putamen, thalamus, amygdala, cerebrum, WM and GM volumes than females $(p<0.05$,

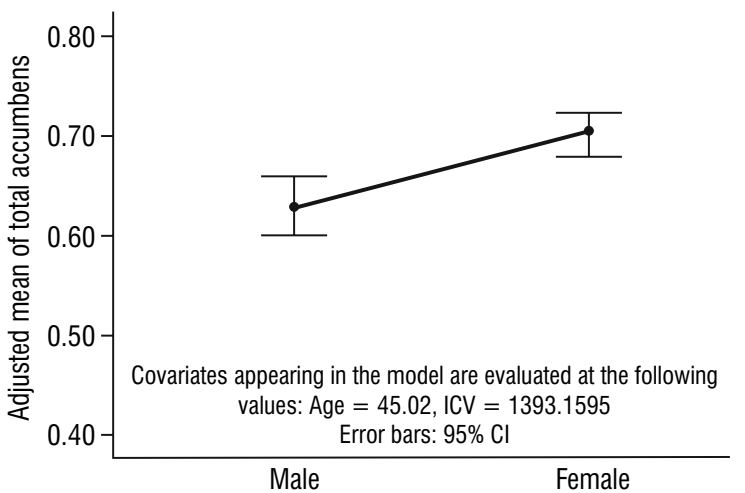

Figure 3. Intracranial volume (ICV)- and age-adjusted mean of total accumbens in males and females; $\mathrm{Cl}$ - confidence interval.

Table 4). However, ICV-adjusted total accumbens mean in females was found to be higher than those in males $(p=0.008)$ (Fig. 5, Table 4). 

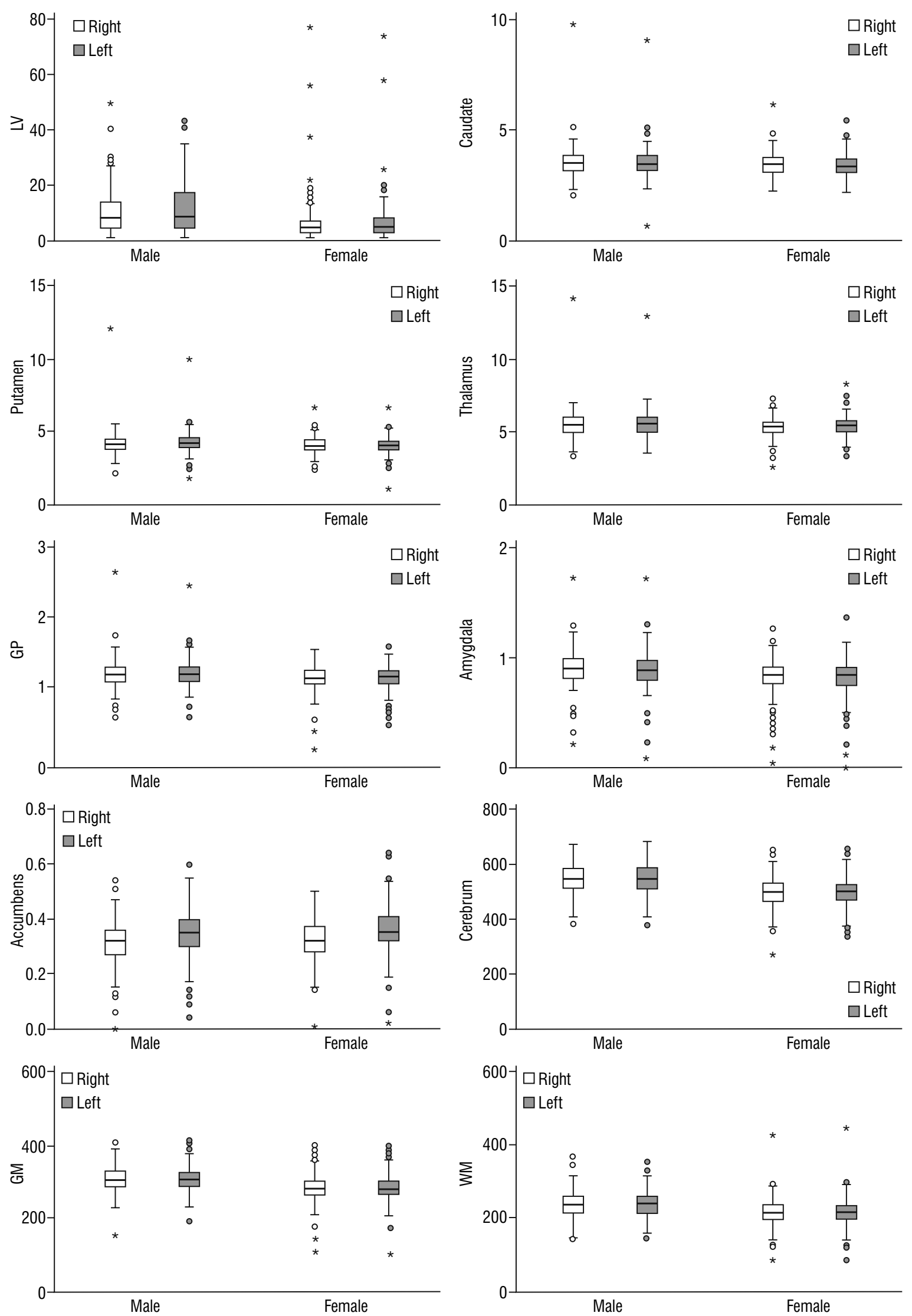

Figure 4. Distribution of right and left sides in males and females; LV — lateral ventricle; GM — grey matter; GP — globus pallidus; WM - white matter. 
Table 3. Distribution of right and left sides in males and females

\begin{tabular}{|c|c|c|c|c|c|}
\hline & \multicolumn{2}{|c|}{ Right } & \multicolumn{2}{|c|}{ Left } & \multirow[t]{2}{*}{$\mathbf{P}$} \\
\hline & Mean \pm SD & Median (min-max) & Mean \pm SD & Median (min-max) & \\
\hline \multicolumn{6}{|l|}{ Male } \\
\hline LV & $10.88 \pm 8.69$ & $8.38(1.26-50.08)$ & $12.20 \pm 9.80$ & $8.71(0.94-43.26)$ & $<0.001$ \\
\hline Caudate & $3.53 \pm 0.80$ & $3.48(1.98-9.84)$ & $3.48 \pm 0.79$ & $3.44(0.61-9.08)$ & 0.010 \\
\hline Putamen & $4.21 \pm 0.94$ & $4.11(2.16-12.05)$ & $4.28 \pm 0.80$ & $4.20(1.89-9.92)$ & $<0.001$ \\
\hline Thalamus & $5.50 \pm 1.13$ & $5.49(3.3-14.18)$ & $5.54 \pm 1.06$ & $5.56(3.49-12.85)$ & 0.086 \\
\hline GP & $1.19 \pm 0.24$ & $1.19(0.57-2.65)$ & $1.20 \pm 0.22$ & $1.19(0.58-2.45)$ & 0.639 \\
\hline Amygdala & $0.90 \pm 0.19$ & $0.90(0.22-1.73)$ & $0.88 \pm 0.20$ & $0.89(0.08-1.72)$ & 0.061 \\
\hline Accumbens & $0.31 \pm 0.09$ & $0.32(0-0.54)$ & $0.35 \pm 0.10$ & $0.35(0.04-0.60)$ & $<0.001$ \\
\hline Cerebrum & $544.06 \pm 61.08$ & $546.91(373.2-671.09)$ & $544.88 \pm 60.04$ & $544.52(373.49-681.72)$ & 0.494 \\
\hline GM & $307.43 \pm 38.41$ & $304.95(154.96-408.61)$ & $306.92 \pm 36.67$ & 305.37 (193.90-413.49) & 0.176 \\
\hline WM & $236.63 \pm 37.87$ & $240.00(145.54-369.85)$ & $237.96 \pm 36.33$ & $240.44(142.55-351.79)$ & 0.010 \\
\hline \multicolumn{6}{|l|}{ Female } \\
\hline LV & $6.35 \pm 7.68$ & $4.61(0.91-77.38)$ & $7.12 \pm 8.35$ & $5.10(0.93-72.55)$ & $<0.001$ \\
\hline Caudate & $3.43 \pm 0.49$ & $3.40(2.19-6.17)$ & $3.39 \pm 0.50$ & $3.33(2.10-5.39)$ & $<0.001$ \\
\hline Putamen & $4.01 \pm 0.53$ & $4.01(2.49-6.69)$ & $4.05 \pm 0.57$ & $4.08(1.08-6.72)$ & $<0.001$ \\
\hline Thalamus & $5.30 \pm 0.65$ & $5.34(2.69-7.30)$ & $5.39 \pm 0.68$ & $5.42(3.33-8.30)$ & $<0.001$ \\
\hline GP & $1.12 \pm 0.18$ & $1.12(0.13-1.54)$ & $1.14 \pm 0.18$ & $1.15(0.46-1.59)$ & 0.028 \\
\hline Amygdala & $0.83 \pm 0.15$ & $0.84(0.04-1.27)$ & $0.82 \pm 0.17$ & $0.84(0.00-1.37)$ & 0.046 \\
\hline Accumbens & $0.32 \pm 0.08$ & $0.32(0.01-0.50)$ & $0.36 \pm 0.09$ & $0.35(0.02-0.64)$ & $<0.001$ \\
\hline Cerebrum & $498.57 \pm 53.79$ & $500.42(269.6-652.79)$ & $498.69 \pm 52.41$ & $499.91(337.56-656.14)$ & 0.689 \\
\hline GM & $282.32 \pm 35.83$ & 279.21 (109.51-398.88) & $281.77 \pm 35.10$ & $279.03(104.76-397.11)$ & 0.012 \\
\hline WM & $216.24 \pm 35.70$ & $214.20(84.72-430.87)$ & $216.92 \pm 35.59$ & $216.41(85.79-448.20)$ & 0.021 \\
\hline
\end{tabular}

SD — standard deviation; LV — lateral ventricle; GP — globus pallidus; GM — grey matter; WM — white matter

\section{Gender differences in the older age group}

In individuals with age greater than 50 years, the univariate analyses resulted in the same as in the younger age group. The ICV-adjusted mean of total thalamus, GP and accumbens volumes were higher in women than in men ( $p<0.05)$ (Fig. 5, Table 4).

When left hemisphere volumes in the younger age group were analysed, males were found to have higher LV, putamen, thalamus, amygdala, cerebrum, WM and GM volumes than females ( $p<0.05$, Table 5 ). However, the ICV-adjusted accumbens mean in females was higher than that in males $(p=0.038)$ (Fig. 6, Table 5). In the older age group, the univariate analyses resulted in the same as in the younger age group. The ICV-adjusted mean of thalamus, GP and accumbens volumes were higher in women than in men ( $p<0.05$ ) (Fig. 6, Table 5).

Right hemisphere volumes in younger and older age groups were almost the same as left hemisphere volumes, except for caudate in the older age groups
(Table 6). The ICV-adjusted mean of caudate was significantly higher in women than in men $(p=0.019)$ (Fig. 7, Table 6).

\section{DISCUSSION}

In our study, we measured the subcortical structures' volumes in healthy adults between the ages of 20-86 and evaluated the data by age and gender.

There are many reports on the brain and subcortical structures' volumes in both sexes and different ages. The majority of these studies focused on the subcortical structures and examined age-related volume changes of various subcortical structures. It has been reported in various cross-sectional and longitudinal studies that the volumes of caudate, thalamus and putamen decrease with age $[6,13,16,29,30$, 32, 36, 39].

Walhovd et al. [36] studied the cortical and subcortical regions of 73 men and women (20-88 years old) using an automated segmentation technique. 
Table 4. Distribution in males and females in younger and older age group ( $\leq 50$ years old and +50 years old in total)

\begin{tabular}{|c|c|c|c|c|c|c|}
\hline & \multicolumn{2}{|r|}{ Male } & \multicolumn{2}{|r|}{ Female } & \multirow[t]{2}{*}{ P-value } & \multirow{2}{*}{$\begin{array}{c}\text { Adj. } \\
\text { p-value }\end{array}$} \\
\hline & Mean \pm SD & Median (min-max) & Mean \pm SD & Median (min-max) & & \\
\hline \multicolumn{7}{|l|}{ Younger age } \\
\hline LV & $15.71 \pm 12.65$ & $11.27(2.20-61.82)$ & $10.84 \pm 13.91$ & 8.35 (2.09-149.93) & 0.001 & 0.301 \\
\hline Caudate & $7.46 \pm 1.82$ & $7.26(4.39-18.92)$ & $7.07 \pm 1.01$ & $6.97(4.92-11.55)$ & 0.122 & 0.563 \\
\hline Putamen & $8.95 \pm 2.03$ & $8.71(5.53-21.97)$ & $8.43 \pm 1.04$ & $8.36(3.60-13.41)$ & 0.030 & 0.803 \\
\hline Thalamus & $11.94 \pm 2.31$ & $11.59(8.87-27.04)$ & $11.18 \pm 1.1$ & $11.05(6.51-14.64)$ & 0.002 & 0.803 \\
\hline GP & $2.48 \pm 0.48$ & $2.42(1.27-5.10)$ & $2.31 \pm 0.38$ & $2.35(0.68-3.14)$ & 0.057 & 0.681 \\
\hline Amygdala & $1.84 \pm 0.3$ & $1.80(0.87-2.60)$ & $1.69 \pm 0.32$ & $1.72(0.04-2.65)$ & $<0.001$ & 0.875 \\
\hline Accumbens & $0.72 \pm 0.18$ & $0.73(0.04-1.08)$ & $0.72 \pm 0.16$ & $0.72(0.07-1.11)$ & 0.769 & 0.008 \\
\hline Cerebrum & $1127.02 \pm 104.65$ & $1114.85(873.65-1345.4)$ & $1029.7 \pm 97.57$ & $1031.47(607.16-1308.94)$ & $<0.001$ & 0.133 \\
\hline WM & $488.91 \pm 64.15$ & $489.75(360.10-701.24)$ & $445.36 \pm 73.99$ & $436.82(170.51-879.07)$ & $<0.001$ & 0.532 \\
\hline GM & $638.11 \pm 79.41$ & $623.75(348.86-814.31)$ & $584.34 \pm 73.66$ & $579.80(214.27-795.99)$ & $<0.001$ & 0.536 \\
\hline \multicolumn{7}{|l|}{ Older age } \\
\hline LV & $31.73 \pm 19.63$ & $27.27(5.91-83.92)$ & $18.1 \pm 18.04$ & $13.04(3.11-114.58)$ & $<0.001$ & 0.068 \\
\hline Caudate & $6.48 \pm 0.89$ & $6.55(4.57-8.77)$ & $6.39 \pm 0.73$ & $6.34(4.41-8.17)$ & 0.551 & 0.072 \\
\hline Putamen & $7.95 \pm 0.94$ & $8.01(5.52-10.13)$ & $7.44 \pm 0.83$ & $7.52(5.08-10.61)$ & 0.002 & 0.594 \\
\hline Thalamus & $9.99 \pm 1.26$ & $9.9(6.86-12.68)$ & $9.82 \pm 1.09$ & $9.99(6.61-11.75)$ & 0.433 & $<0.001$ \\
\hline GP & $2.28 \pm 0.36$ & $2.3(1.48-3.38)$ & $2.17 \pm 0.24$ & $2.17(1.53-2.67)$ & 0.066 & 0.037 \\
\hline Amygdala & $1.7 \pm 0.38$ & $1.69(0.44-2.52)$ & $1.56 \pm 0.28$ & $1.61(0.72-2.06)$ & 0.013 & 0.123 \\
\hline Accumbens & $0.59 \pm 0.14$ & $0.60(0.12-0.87)$ & $0.61 \pm 0.14$ & $0.63(0.34-1.11)$ & 0.502 & 0.007 \\
\hline Cerebrum & $1044.27 \pm 121$ & $1058.10(746.69-1328.12)$ & $942.47 \pm 99.16$ & $939.27(712.33-1173.36)$ & $<0.001$ & 0.176 \\
\hline WM & $457.8 \pm 79.79$ & $452.80(288.09-630.98)$ & $413.88 \pm 64.73$ & $408.89(245.55-582.04)$ & 0.001 & 0.096 \\
\hline GM & $586.47 \pm 56.6$ & $579.86(458.6-740.8)$ & $528.59 \pm 48.23$ & $534.87(350.09-638.26)$ & $<0.001$ & 0.969 \\
\hline
\end{tabular}

Bold measurements are significantly higher in females than those in males after total intracranial volume adjustment. SD — standard deviation; LV — lateral ventricle; GP — globus pallidus; WM — white matter; GM — grey matter; Adj. p-value — p-value after total intracranial volume adjustment

Except for pallidum, they showed age-related volume decrease in cortical grey matter, cerebral white matter, hippocampus, amygdala, thalamus, accumbens, caudate, putamen, pallidus, brainstem, cerebellar cortex and cerebellar white matter. They also found that advanced age was strongly associated with volumes of the thalamus and cortical grey matter, which showed a linear decline and curvilinear decline with age.

In our study, total thalamus, GP and accumbens volumes in patients older than 50 years were higher in women. In our findings, we did not find an age-related decrease in left thalamus volume in a similar age range (20-86 years) and a higher thalamus volume in men over the age of 50 years.

Alexander et al. [3] stated that putamen, caudate, accumbens and pallidum, all of which are related to emotional, motor behaviour and cognition, are blunted by aging. Basal ganglia reach a peak volume before the age of 20 . The youngest participant in our study was people aged 11-84 years were evaluated. Therefore, the basal ganglia did not go through an atrophy period.

Accumbens and pallidum volumes, which are reported as less stable in the literature than other subcortical structures [16], showed a minimal age effect on pallidum than other structures. Walhovd et al. [37] examined the effects of age on subcortical structures and they found great differences in putamen, thalamus and accumbens volume due to aging. Regions such as caudate and amygdala were not affected by aging.

In the subcortical regions such as caudate, pallidum and amygdala, a linear decrease in the age-related pattern is shown [13, 29, 30]. Pfefferbaum et al. [27] performed MRI studies in 55 men and 67 women (20-85 years), and showed that older age was related to a decrease in thalamus volume, and this decline increased with age (60+ years). 

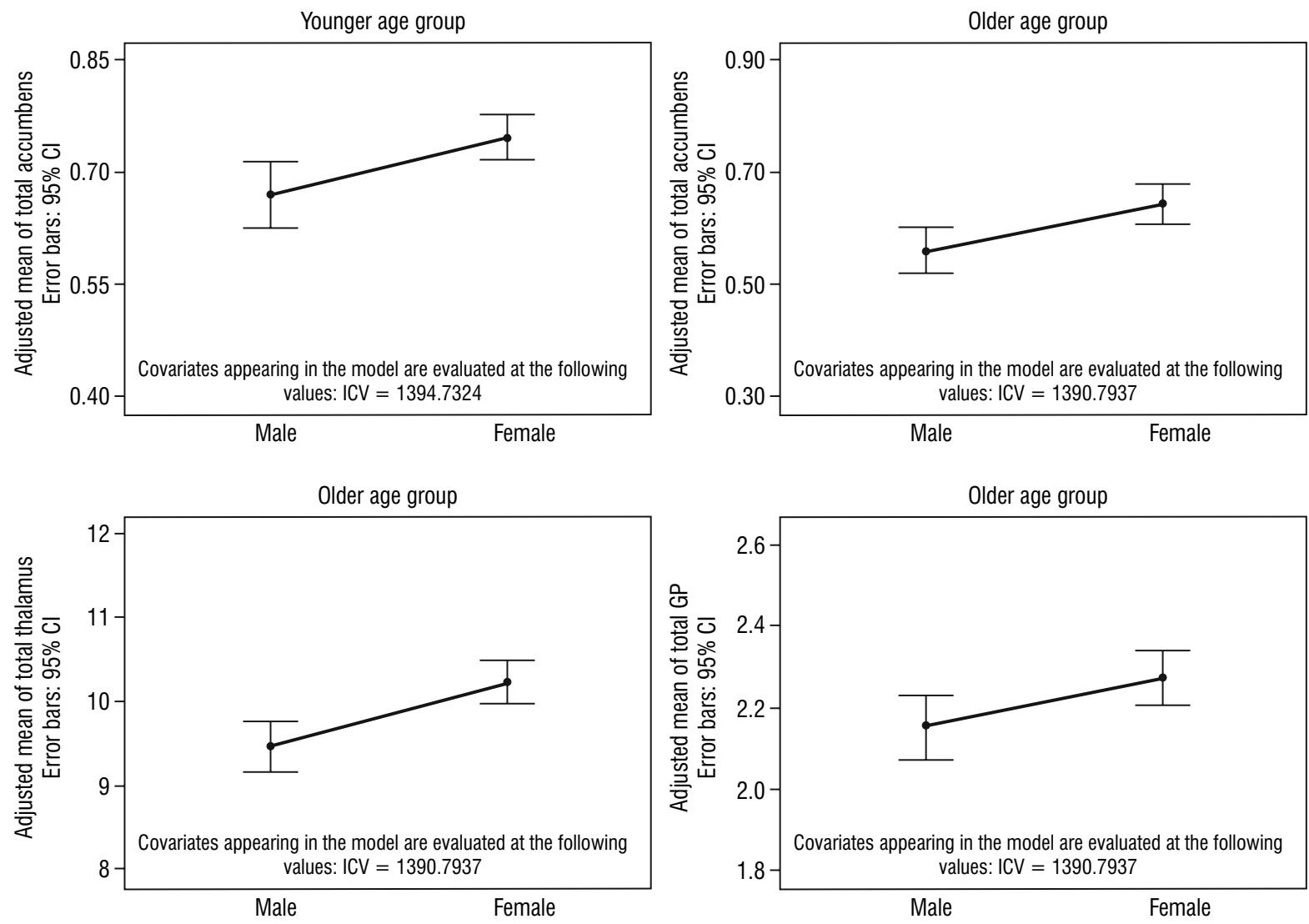

Figure 5. Intracranial volume (ICV)-adjusted mean of total accumbens, thalamus and globus pallidus (GP) volumes in males and females of younger and older age groups; $\mathrm{Cl}$ - confidence interval.

Abedalahi and Hasanzadeh [1] calculated the caudate volume with the Cavalieri principle. This study was carried out in 120 normal human subjects (60 males, 60 females) divided into young ( $<40$ years) and older $(\geq 40$ years) groups between the ages of $15-65$. The volume of caudate nucleus showed a significant negative correlation with age. Goodre et al. [16] showed that there was a stronger correlation between the age and the structural volume for the hippocampus, amygdala and accumbens in the older group (60-85 years) than the middle-aged (35-60 years) group. Similarly, in our study, 303 normal people (113 men, 190 women) aged 11-84 were divided into young $(\leq 50$ years old) and elderly ( $>50$ years old) groups. There was a significant negative correlation between the young group with the volume of the accumbens and the old group with the volume of the thalamus, GP, and accumbens.

Thalamic volumetric analyses showed that thalamus volumes were smaller in the elderly than younger adults using cross-sectional and automated technique studies [6,36]. Cherubini et al. [6] found that age showed strong correlation in both striatal structures and thalamic volume. In our study, gender differences in cortical grey matter concentration were greater in patients aged 50 years and older than in the younger age group; LV, putamen, thalamus, amygdala, cerebrum, WM and GM volumes of men were higher than women. However, women had significantly higher accumbens volume. Unlike our findings, Wang et al. [39] stated in their study that the volume of accumbens decreased with increasing age. The effect of gender on the volume of subcortical structures may play an important role because basal ganglia have high-density sex steroid receptors [34]. In this period, age and gender interaction showed that the right putamen and right pallidum in men had a marked age progression.

In general, male brains were found to have larger GM, WM, and subcortical structures than women. As a result of the correction of the total intracranial volume, we found that women had a larger volume of accumbens. In addition, in the male group only, we found a significant large volume effect on the 
Table 5. Distribution in males and females in younger and older age group ( $\leq 50$ years old and +50 years old) in left hemisphere

\begin{tabular}{|c|c|c|c|c|c|c|}
\hline \multirow{2}{*}{$\begin{array}{l}\text { Left } \\
\text { hemisphere }\end{array}$} & \multicolumn{2}{|c|}{ Male } & \multicolumn{2}{|c|}{ Female } & \multirow[t]{2}{*}{ P-value } & \multirow{2}{*}{$\begin{array}{c}\text { Adj. } \\
\text { p-value }\end{array}$} \\
\hline & Mean \pm SD & Median (min-max) & Mean \pm SD & Median (min-max) & & \\
\hline \multicolumn{7}{|l|}{ Younger age } \\
\hline LV & $8.37 \pm 7.31$ & $5.61(0.94-35.01)$ & $5.65 \pm 6.89$ & $4.40(0.93-72.55)$ & 0.003 & 0.256 \\
\hline Caudate & $3.69 \pm 0.94$ & $3.61(0.61-9.08)$ & $3.53 \pm 0.50$ & $3.49(2.53-5.39)$ & 0.119 & 0.520 \\
\hline Putamen & $4.50 \pm 0.94$ & $4.43(1.89-9.92)$ & $4.23 \pm 0.56$ & $4.19(1.08-6.72)$ & 0.008 & 0.800 \\
\hline Thalamus & $5.99 \pm 1.15$ & $5.91(3.49-12.85)$ & $5.65 \pm 0.60$ & $5.63(3.82-8.30)$ & 0.003 & 0.947 \\
\hline GP & $1.24 \pm 0.24$ & $1.20(0.58-2.45)$ & $1.17 \pm 0.19$ & $1.20(0.46-1.59)$ & 0.167 & 0.464 \\
\hline Amygdala & $0.91 \pm 0.19$ & $0.91(0.08-1.31)$ & $0.84 \pm 0.18$ & $0.86(0-1.37)$ & $<0.001$ & 0.900 \\
\hline Accumbens & $0.38 \pm 0.10$ & $0.37(0.04-0.60)$ & $0.38 \pm 0.09$ & $0.37(0.02-0.63)$ & 0.578 & 0.038 \\
\hline Cerebrum & $563.46 \pm 53.99$ & 555.28 (434.27-681.72) & $514.43 \pm 47.27$ & $514.91(337.56-656.14)$ & $<0.001$ & 0.219 \\
\hline WM & $244.64 \pm 31.60$ & $245.31(179.41-351.79)$ & $222.43 \pm 36.28$ & 219.81 (85.79-448.20) & $<0.001$ & 0.651 \\
\hline GM & $318.82 \pm 39.49$ & $310.69(193.90-413.49)$ & $291.99 \pm 36.38$ & $290.19(104.76-397.11)$ & $<0.001$ & 0.554 \\
\hline \multicolumn{7}{|l|}{ Older age } \\
\hline LV & $16.70 \pm 10.48$ & $14.91(2.38-43.26)$ & $9.69 \pm 9.98$ & $6.44(1.34-58.48)$ & $<0.001$ & 0.088 \\
\hline Caudate & $3.23 \pm 0.46$ & $3.27(2.29-4.33)$ & $3.16 \pm 0.39$ & $3.12(2.10-4.27)$ & 0.393 & 0.227 \\
\hline Putamen & $4.02 \pm 0.50$ & $4.04(2.51-5.16)$ & $3.73 \pm 0.42$ & $3.77(2.58-5.26)$ & 0.001 & 0.857 \\
\hline Thalamus & $5.02 \pm 0.64$ & $4.95(3.53-6.38)$ & $4.93 \pm 0.56$ & $4.99(3.33-6.11)$ & 0.440 & 0.002 \\
\hline GP & $1.15 \pm 0.19$ & $1.14(0.72-1.66)$ & $1.09 \pm 0.13$ & $1.09(0.67-1.32)$ & 0.046 & 0.047 \\
\hline Amygdala & $0.83 \pm 0.21$ & $0.82(0.22-1.72)$ & $0.78 \pm 0.15$ & $0.79(0.20-1.01)$ & 0.082 & 0.168 \\
\hline Accumbens & $0.31 \pm 0.08$ & $0.32(0.09-0.53)$ & $0.33 \pm 0.08$ & $0.33(0.19-0.64)$ & 0.423 & 0.016 \\
\hline Cerebrum & $523.09 \pm 59.90$ & 532.08 (373.49-662.49) & $471.08 \pm 49.77$ & $471.04(350.15-588.37)$ & $<0.001$ & 0.301 \\
\hline WM & $230.14 \pm 40.10$ & $227.97(142.55-316.04)$ & $207.24 \pm 32.37$ & $205.90(122.11-292.40)$ & 0.001 & 0.126 \\
\hline GM & $292.95 \pm 27.39$ & $289.34(230.94-371.23)$ & $263.84 \pm 24.02$ & $265.02(171.92-317.10)$ & $<0.001$ & 0.675 \\
\hline
\end{tabular}

Bold measurements are significantly higher in females than those in males after total intracranial volume adjustment. SD — standard deviation; LV — lateral ventricle; GP — globus pallidus; WM — white matter; GM — grey matter; Adj. p-value — p-value after total intracranial volume adjustment

right caudate volume compared to the contralateral structures of the brain hemisphere.

Ruigrok et al. [32] found that the TB volume was on average $8-15 \%$ higher in males than females. Wang et al. [39] reported that the volume of the right putamen, right pallidum, and right thalamus decreased faster in males, whereas the volume of the left thalamus, bilateral hippocampus and amygdala follow a quadratic model in males and a linear decline model in females. In our study, in men, LV, putamen, accumbens and WM volumes in the right brain were significantly lower than the left brain and higher than the left brain for caudate volume.

Xu et al. [40] found that males had atrophy due to aging in the basal ganglia of the left hemisphere and also the volume of the thalamus and brain in the left hemisphere was significantly smaller than the right hemisphere. Brain atrophy with aging in male patients was higher than female patients. We found left hemisphere volumes in males from both younger and older age group were higher for LV, putamen, thalamus, amygdala, cerebrum, WM and GM volumes than in females. However, accumbens volume mean in females was higher than that in males. Nevertheless, right hemisphere volumes in younger and older age groups were almost the same as left hemisphere volumes, except caudate in the older age group. The mean volume of caudate was significantly higher in women than in men.

Goodro et al. [16] found that left and right thalamus volume decreased faster than women. Similarly, in our study, the volumes of thalamus in women and men were different in the left and right thalamus. In women between the ages of 20-86, the left thalamic volume was higher than in men.

Although our MRI research cannot identify the mechanism that leads to volume differences due to age, it can provide additional information for the 

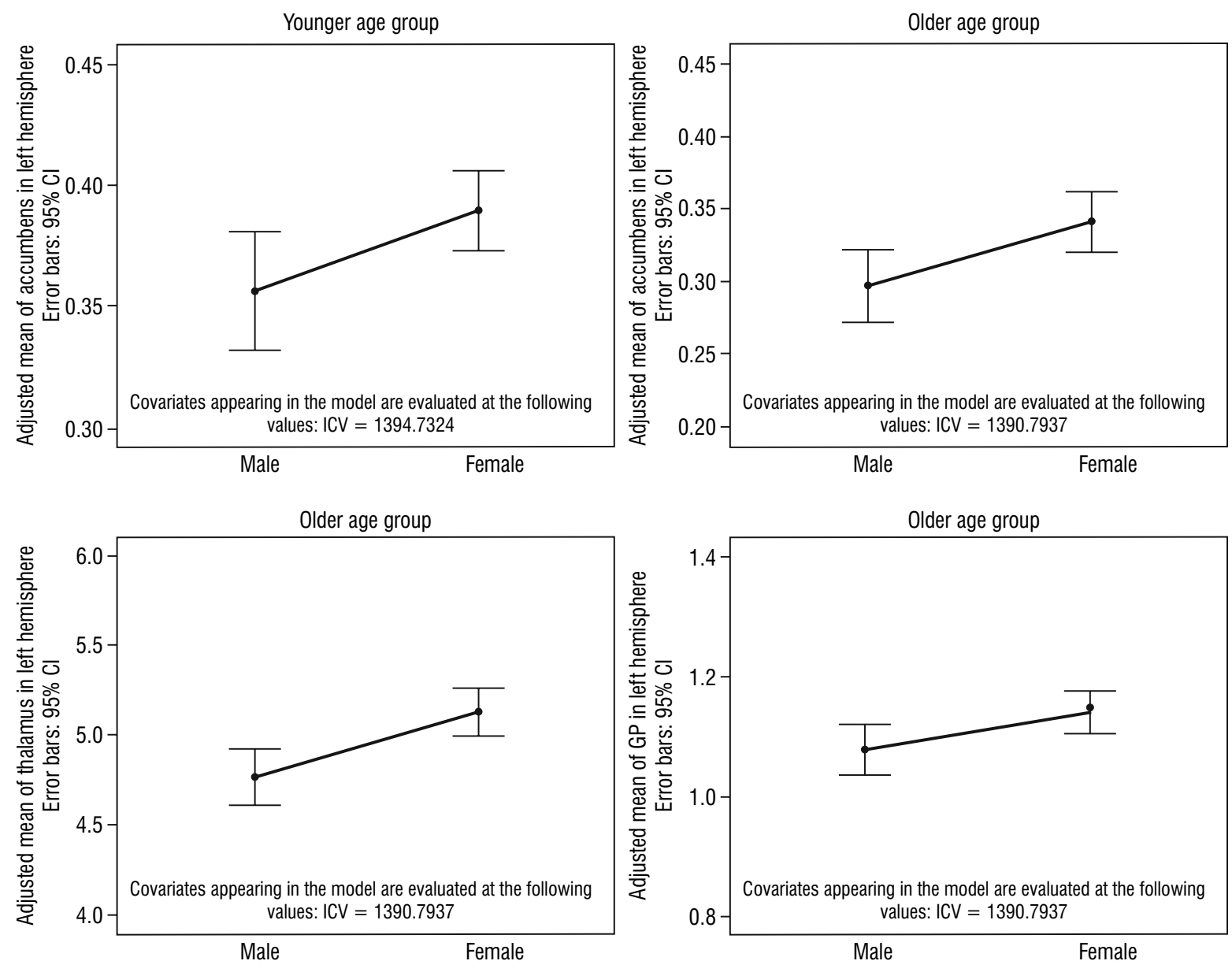

Figure 6. Intracranial volume (ICV)-adjusted mean of left hemisphere accumbens, thalamus and globus pallidus (GP) volumes in males and females of younger and older age groups; $\mathrm{Cl}$ - confidence interval.

possible mechanism of sex-dependent volume differences in basal ganglia when associated with advanced software technology. Volume analysis of the nucleus accumbens helps in the evaluation of neurodegenerative diseases. In this context, our results are of importance for gender-dependent nucleus accumbens volume increase.

Compared to women, men have been found to perform worse in the ongoing response task and visual-spatial learning and planning task, especially in older ages $[7,28]$. However, some studies have not reported age and gender effects or different aging effects on cognition in men and women [20,33].

In this study, we used an automated and reliable analysis to address volumetric changes [23]. We presented a new method, namely, volBrain. It can be used in place of other volume techniques. volBrain has several advantages for brain imaging researchers. In addition, it is less tiring software that can get very fast results in the treatments and clinical studies in neurological disorders.

The main limitation of our study is that it is not a longitudinal study. More participants and more studies are needed to confirm the findings of this study. Another limitation is that it is not done using manual volumetry.

volBrain can be used to measure volumes of other anatomical areas of the body using radiological images. We believe that our results will provide additional information to volumetric studies evaluating the development, pathology and abnormalities of subcortical structures.

The results of the study demonstrated that in the age group of 50 years or less, males were found to have higher total LV, putamen, thalamus, amygdala, cerebrum, WM and GM volumes than females. In the age group over 50 years, the means of total thalamus, GP, and accumbens volumes were higher in females than those in males. 
Table 6. Distribution in males and females in younger and older age group ( $\leq 50$ years old and +50 years old) in right hemisphere

\begin{tabular}{|c|c|c|c|c|c|c|}
\hline \multirow{2}{*}{$\begin{array}{l}\text { Right } \\
\text { hemisphere }\end{array}$} & \multicolumn{2}{|r|}{ Male } & \multicolumn{2}{|c|}{ Female } & \multirow[t]{2}{*}{$\mathrm{p}$-value } & \multirow{2}{*}{$\begin{array}{c}\text { Adj. } \\
\text { p-value }\end{array}$} \\
\hline & Mean \pm SD & Median (min-max) & Mean \pm SD & Median (min-max) & & \\
\hline \multicolumn{7}{|l|}{ Younger age } \\
\hline LV & $7.34 \pm 5.72$ & $5.50(1.26-28.33)$ & $5.19 \pm 7.12$ & $4.19(0.91-77.38)$ & 0.001 & 0.370 \\
\hline Caudate & $3.77 \pm 0.95$ & $3.70(1.98-9.84)$ & $3.55 \pm 0.52$ & $3.51(2.19-6.17)$ & 0.055 & 0.635 \\
\hline Putamen & $4.45 \pm 1.16$ & $4.29(2.16-12.05)$ & $4.19 \pm 0.50$ & $4.14(2.52-6.69)$ & 0.052 & 0.812 \\
\hline Thalamus & $5.95 \pm 1.25$ & $5.78(3.60-14.18)$ & $5.53 \pm 0.58$ & $5.51(2.69-7.30)$ & $<0.001$ & 0.686 \\
\hline GP & $1.24 \pm 0.26$ & $1.20(0.59-2.65)$ & $1.14 \pm 0.20$ & $1.14(0.13-1.54)$ & 0.015 & 0.907 \\
\hline Amygdala & $0.93 \pm 0.14$ & $0.90(0.47-1.29)$ & $0.85 \pm 0.16$ & $0.86(0.04-1.27)$ & 0.001 & 0.857 \\
\hline Accumbens & $0.34 \pm 0.09$ & $0.34(0-0.54)$ & $0.35 \pm 0.08$ & $0.34(0.01-0.50)$ & 0.859 & 0.002 \\
\hline Cerebrum & $563.56 \pm 53.68$ & $559.56(413.08-671.09)$ & $514.07 \pm 49.95$ & $515.50(269.60-652.79)$ & $<0.001$ & 0.220 \\
\hline WM & $244.28 \pm 34.36$ & $245.42(151.33-369.85)$ & $221.72 \pm 36.41$ & $220.98(84.72-430.87)$ & $<0.001$ & 0.653 \\
\hline GM & $319.28 \pm 41.18$ & $317.76(154.96-408.61)$ & $292.35 \pm 37.48$ & 289.57 (109.51-398.88) & $<0.001$ & 0.528 \\
\hline \multicolumn{7}{|l|}{ Older age } \\
\hline LV & $15.03 \pm 9.73$ & $12.58(3.31-50.08)$ & $8.39 \pm 8.26$ & $6.04(1.54-56.10)$ & $<0.001$ & 0.058 \\
\hline Caudate & $3.25 \pm 0.45$ & $3.23(2.25-4.44)$ & $3.22 \pm 0.35$ & $3.20(2.31-4.01)$ & 0.697 & 0.019 \\
\hline Putamen & $3.93 \pm 0.46$ & $3.98(2.91-4.97)$ & $3.70 \pm 0.43$ & $3.70(2.49-5.35)$ & 0.005 & 0.383 \\
\hline Thalamus & $4.97 \pm 0.67$ & $4.92(3.30-6.31)$ & $4.89 \pm 0.55$ & $4.99(3.28-5.75)$ & 0.651 & 0.001 \\
\hline GP & $1.14 \pm 0.19$ & $1.15(0.57-1.73)$ & $1.09 \pm 0.12$ & $1.09(0.82-1.35)$ & 0.058 & 0.046 \\
\hline Amygdala & $0.87 \pm 0.22$ & $0.86(0.22-1.73)$ & $0.79 \pm 0.14$ & $0.81(0.30-1.05)$ & 0.025 & 0.181 \\
\hline Accumbens & $0.27 \pm 0.07$ & $0.28(0-0.42)$ & $0.28 \pm 0.07$ & $0.29(0.14-0.48)$ & 0.660 & 0.005 \\
\hline Cerebrum & $521.18 \pm 61.75$ & $527.69(373.20-665.65)$ & $471.39 \pm 49.61$ & 471.10 (362.18-584.99) & $<0.001$ & 0.098 \\
\hline WM & $227.66 \pm 40.11$ & $224.25(145.54-314.94)$ & $206.64 \pm 32.48$ & 204.41 (123.44-289.64) & 0.002 & 0.075 \\
\hline GM & $293.52 \pm 29.65$ & $290.40(227.66-369.58)$ & $264.75 \pm 24.42$ & 268.09 (178.17-321.15) & $<0.001$ & 0.729 \\
\hline
\end{tabular}

Bold measurements are significantly higher in females than those in males after total intracranial volume adjustment. SD — standard deviation; LV — lateral ventricle; GP — globus pallidus; WM — white matter; GM — grey matter; Adj. p-value — p-value after total intracranial volume adjustment

\section{CONCLUSIONS}

In conclusion, the results of recent research show that brain cortical structures and volume loss of subcortical nuclei are a common finding in a number of neuropsychiatric problems. The data obtained in this study are normal brain data according to age and gender of the adult Turkish population. It can be useful in clinical applications and cognitive disorders of many neuropsychiatric diseases.

\section{Acknowledgements}

The authors thank the Department of Radiology of Dışkapı Training and Research Hospital for providing the raw data, the Ethics Committee for approving the permits, all contributing authors, and the faculty members of the Department of Foreign Languages, Erciyes University for the English editing, to Ankara Yıldırım Beyazıt University Department of Biostatistics for statistical analysis.
Conflict of interest: None declared

\section{REFERENCES}

1. Abedalahi A, Hasanzadeh H. MRI based morphometry of caudate nucleus in normal. Persons. Archives of advances in biosciences. J Paramedical Sci. 2013; 42(2): 70-75.

2. Acer N, Bastepe-Gray S, Sagiroglu A, et al. Diffusion tensor and volumetric magnetic resonance imaging findings in the brains of professional musicians. J Chem Neuroanat. 2018; 88: 33-40, doi: 10.1016/j.jchemneu.2017.11.003, indexed in Pubmed: 29113947.

3. Alexander GE, DeLong MR, Strick PL. Parallel organization of functionally segregated circuits linking basal ganglia and cortex. Annu Rev Neurosci. 1986; 9: 357-381, doi: 10.1146/annurev.ne.09.030186.002041, indexed in Pubmed: 3085570.

4. Allen JS, Bruss J, Brown CK, et al. Normal neuroanatomical variation due to age: the major lobes and a parcellation of the temporal region. Neurobiol Aging. 2005; 26(9): 1245-1260, doi: 10.1016/j.neurobiolaging.2005.05.023, indexed in Pubmed: 16046030.

5. Bourque M, Dluzen DE, Di Paolo T. Neuroprotective actions of sex steroids in Parkinson's disease. Front 

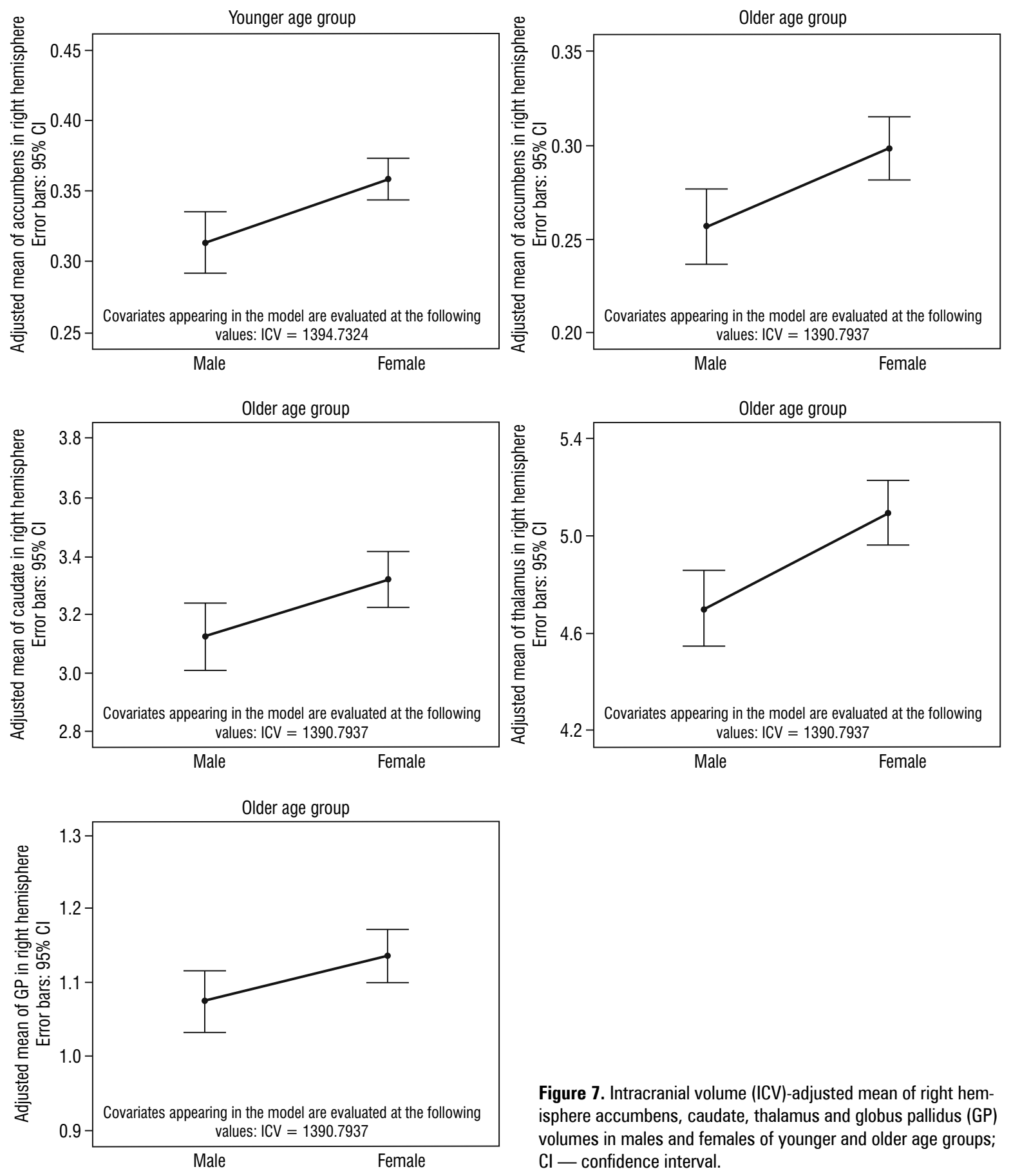

Figure 7. Intracranial volume (ICV)-adjusted mean of right hemisphere accumbens, caudate, thalamus and globus pallidus (GP) volumes in males and females of younger and older age groups; $\mathrm{Cl}$ - confidence interval.

Neuroendocrinol. 2009; 30(2): 142-157, doi: 10.1016/j. yfrne.2009.04.014, indexed in Pubmed: 19410597.

6. Cherubini A, Péran P, Caltagirone $C$, et al. Aging of subcortical nuclei: microstructural, mineralization and atrophy modifications measured in vivo using MRI. Neuroimage. 2009; 48(1): 29-36, doi: 10.1016/j.neuroimage.2009.06.035, indexed in Pubmed: 19540925.

7. Clark $C R$, Paul RH, Williams LM, et al. Standardized assessment of cognitive functioning during development and aging using an automated touchscreen battery. Arch
Clin Neuropsychol. 2006; 21(5): 449-467, doi: 10.1016/j. acn.2006.06.005, indexed in Pubmed: 16904862.

8. Corson PW, Nopoulos P, Miller DD, et al. Change in basal ganglia volume over 2 years in patients with schizophrenia: typical versus atypical neuroleptics. Am J Psychiatry. 1999; 156(8): 1200-1204, doi: 10.1176/ajp.156.8.1200, indexed in Pubmed: 10450260.

9. Coupé P, Manjón JV, Fonov V, et al. Patch-based segmentation using expert priors: application to hippocampus and ventricle segmentation. Neuroimage. 2011; 54(2): 
940-954, doi: 10.1016/j.neuroimage.2010.09.018, indexed in Pubmed: 20851199.

10. Cox RW. AFNI: what a long strange trip it's been. Neuroimage. 2012; 62(2): 743-747, doi: 10.1016/j.neuroimage.2011.08.056, indexed in Pubmed: 21889996.

11. Filipek PA, Richelme C, Kennedy DN, et al. The young adult human brain: an MRI-based morphometric analysis. Cereb Cortex. 1994; 4(4): 344-360, doi: 10.1093/cercor/4.4.344, indexed in Pubmed: 7950308.

12. Fischl B. FreeSurfer. Neuroimage. 2012; 62(2): 774-781, doi: 10.1016/j.neuroimage.2012.01.021, indexed in Pubmed: 22248573.

13. Fjell AM, Westlye LT, Grydeland $H$, et al. Critical ages in the life course of the adult brain: nonlinear subcortical aging. Neurobiol Aging. 2013; 34(10): 2239-2247, doi: 10.1016/j.neurobiolaging.2013.04.006, indexed in Pubmed: 23643484

14. Gilbert A, Keshavan M. MRI structural findings in schizophrenia. Revista Brasileira de Psiquiatria. 2001; 23(suppl 1): 15-18, doi: 10.1590/s1516-44462001000500006.

15. Goebel R. BrainVoyager -- past, present, future. Neuroimage. 2012; 62(2): 748-756, doi: 10.1016/j.neuroimage.2012.01.083, indexed in Pubmed: 22289803.

16. Goodro M, Sameti M, Patenaude B, et al. Age effect on subcortical structures in healthy adults. Psychiatry Res. 2012; 203(1): 38-45, doi: 10.1016/j.pscychresns.2011.09.014, indexed in Pubmed: 22863654.

17. Huang $X, P u$ W, Li $X$, et al. Decreased left putamen and thalamus volume correlates with delusions in first-episode schizophrenia patients. Front Psychiatry. 2017; 8: 245, doi: 10.3389/fpsyt.2017.00245, indexed in Pubmed: 29209237.

18. Igual L, Soliva JC, Hernández-Vela A, et al. A fully-automatic caudate nucleus segmentation of brain MRI: application in volumetric analysis of pediatric attention-deficit/hyperactivity disorder. Biomed Eng Online. 2011; 10: 105, doi: 10.1186/1475-925X-10-105, indexed in Pubmed: 22141926.

19. Jiji S, Smitha KA, Gupta AK, et al. Segmentation and volumetric analysis of the caudate nucleus in Alzheimer's disease. Eur J Radiol. 2013; 82(9): 1525-1530, doi: 10.1016/j. ejrad.2013.03.012, indexed in Pubmed: 23664648.

20. Kavé G, Shrira A, Palgi Y, et al. Formal education level versus self-rated literacy as predictors of cognitive aging. J Gerontol B Psychol Sci Soc Sci. 2012; 67(6): 697-704, doi: 10.1093/geronb/gbs031, indexed in Pubmed: 22421808.

21. Kocaman H, Acer N, Köseoğlu E, et al. Evaluation of intracerebral ventricles volume of patients with Parkinson's disease using the atlas-based method: A methodological study. J Chem Neuroanat. 2019; 98: 124-130, doi: 10.1016/j. jchemneu.2019.04.005, indexed in Pubmed: 30986488.

22. Madan CR, Kensinger EA. Age-related differences in the structural complexity of subcortical and ventricular structures. Neurobiol Aging. 2017; 50: 87-95, doi: 10.1016/j.neurobiolaging.2016.10.023, indexed in Pubmed: 27939959.

23. Manjón JV, Coupé P. volBrain: An Online MRI brain volumetry system. Front Neuroinform. 2016; 10: 30, doi: 10.3389/ fninf.2016.00030, indexed in Pubmed: 27512372.

24. Morey RA, Petty CM, Xu Y, et al. A comparison of automated segmentation and manual tracing for quantifying hippocampal and amygdala volumes. Neuroimage. 2009; 45(3): 855-866, doi: 10.1016/j.neuroimage.2008.12.033, indexed in Pubmed: 19162198.

25. Morey RA, Selgrade ES, Wagner HR, et al. Scan-rescan reliability of subcortical brain volumes derived from automated segmentation. Hum Brain Mapp. 2010; 31(11): 1751-1762, doi: 10.1002/hbm.20973, indexed in Pubmed: 20162602.

26. Palancı Ö, Kalaycıoğlu A, Acer N, et al. Volume calculation of brain structures in migraine disease by using mristudio. NeuroQuantology. 2018; 16(10): 8-13, doi: 10.14704/ nq.2018.16.10.1692.
27. Pfefferbaum A, Rohlfing T, Rosenbloom MJ, et al. Variation in longitudinal trajectories of regional brain volumes of healthy men and women (ages 10 to 85 years) measured with atlas-based parcellation of MRI. Neuroimage. 2013; 65: 176-193, doi: 10.1016/j.neuroimage.2012.10.008, indexed in Pubmed: 23063452.

28. Proust-Lima C, Amieva H, Letenneur L, et al. Gender and education impact on brain aging: a general cognitive factor approach. Psychol Aging. 2008; 23(3): 608-620, doi: 10.1037/a0012838, indexed in Pubmed: 18808250.

29. Raz N, Lindenberger U. Only time will tell: cross-sectional studies offer no solution to the age-brain-cognition triangle: comment on Salthouse (2011). Psychol Bull. 2011; 137(5): 790-795, doi: 10.1037/a0024503, indexed in Pubmed: 21859179.

30. Raz N, Lindenberger U, Rodrigue KM, et al. Regional brain changes in aging healthy adults: general trends, individual differences and modifiers. Cereb Cortex. 2005; 15(11): 1676-1689, doi: 10.1093/cercor/bhi044, indexed in Pubmed: 15703252.

31. Rijpkema M, Everaerd D, van der Pol C, et al. Normal sexual dimorphism in the human basal ganglia. Hum Brain Mapp. 2012; 33(5): 1246-1252, doi: 10.1002/hbm.21283, indexed in Pubmed: 21523857.

32. Ruigrok ANV, Salimi-Khorshidi G, Lai MC, et al. A meta-analysis of sex differences in human brain structure. Neurosci Biobehav Rev. 2014; 39: 34-50, doi: 10.1016/j. neubiorev.2013.12.004, indexed in Pubmed: 24374381.

33. Silver H, Goodman C, Gur RC, et al. 'Executive' functions and normal aging: selective impairment in conditional exclusion compared to abstraction and inhibition. Dement Geriatr Cogn Disord. 2011; 31(1): 53-62, doi: 10.1159/000322568, indexed in Pubmed: 21150204.

34. Taber KH, Murphy DD, Blurton-Jones MM, et al. An update on estrogen: higher cognitive function, receptor mapping, neurotrophic effects. J Neuropsychiatry Clin Neurosci. 2001; 13(3): 313-317, doi: 10.1176/jnp.13.3.313, indexed in Pubmed: 11514636.

35. Volkow ND, Wang GJ, Fowler JS, et al. Addiction circuitry in the human brain. Annu Rev Pharmacol Toxicol. 2012; 52: 321-336, doi: 10.1146/annurev-pharmtox-010611-134625, indexed in Pubmed: 21961707.

36. Walhovd KB, Fjell AM, Reinvang I, et al. Effects of age on volumes of cortex, white matter and subcortical structures. Neurobiol Aging. 2005; 26(9): 1261-1270, doi: 10.1016/j.neurobiolaging.2005.05.020, indexed in Pubmed: 16005549.

37. Walhovd KB, Westlye LT, Amlien I, et al. Consistent neuroanatomical age-related volume differences across multiple samples. Neurobiol Aging. 2011; 32(5): 916-932, doi: 10.1016/j.neurobiolaging.2009.05.013, indexed in Pubmed: 19570593.

38. Wang $Y, X u Q$, Li S, et al. Gender differences in anomalous subcortical morphology for children with ADHD. Neurosci Lett. 2018; 665: 176-181, doi: 10.1016/j.neulet.2017.12.006, indexed in Pubmed: 29217259.

39. Wang $Y, X u Q$, Luo J, et al. Effects of age and sex on subcortical volumes. Front Aging Neurosci. 2019; 11(259): 1-12, doi: 10.3389/fnagi.2019.00259, indexed in Pubmed: 31616285.

40. Xu J, Kobayashi S, Yamaguchi S, et al. Gender effects on age-related changes in brain structure. AJNR Am J Neuroradiol. 2000; 21(1): 112-118.

41. Zheng $F$, Li C, Zhang $D$, et al. Study on the sub-regions volume of hippocampus and amygdala in schizophrenia. Quant Imaging Med Surg. 2019; 9(6): 1025-1036, doi: 10.21037/qims.2019.05.21, indexed in Pubmed: 31367556 . 